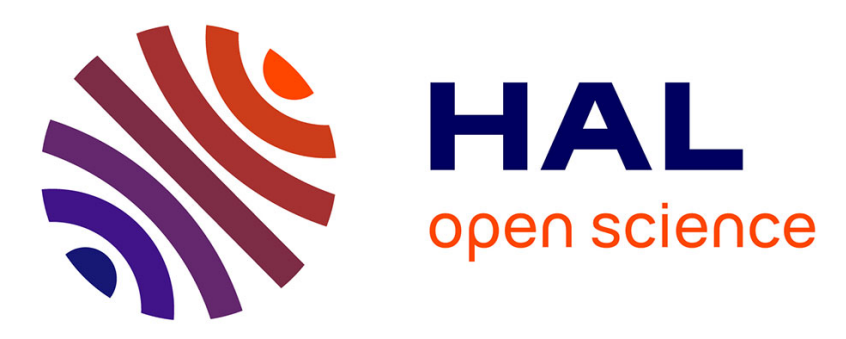

\title{
Des fonctions du double chez Rank et Freud à la romance amoureuse à l'adolescence
}

\author{
Julie Chevalier, Bonnet Christian, Guy Gimenez
}

\section{To cite this version:}

Julie Chevalier, Bonnet Christian, Guy Gimenez. Des fonctions du double chez Rank et Freud à la romance amoureuse à l'adolescence. Bulletin de psychologie, 2020, 565, pp.33-42. 10.3917/bupsy.565.0033 . hal-02476522

\section{HAL Id: hal-02476522 \\ https://hal-amu.archives-ouvertes.fr/hal-02476522}

Submitted on 12 Feb 2020

HAL is a multi-disciplinary open access archive for the deposit and dissemination of scientific research documents, whether they are published or not. The documents may come from teaching and research institutions in France or abroad, or from public or private research centers.
L'archive ouverte pluridisciplinaire HAL, est destinée au dépôt et à la diffusion de documents scientifiques de niveau recherche, publiés ou non, émanant des établissements d'enseignement et de recherche français ou étrangers, des laboratoires publics ou privés. 


\title{
Des fonctions du double chez Rank et Freud à la romance amoureuse à l'adolescence
}

\author{
Chevalier Julie ${ }^{a}$ \\ BONNET Christian ${ }^{\text {a }}$ \\ Gimenez Guy ${ }^{a}$
}

a Aix Marseille Université, Laboratoire de psychologie clinique, de psychopathologie et de psychanalayse, (LPCPP), Aix-en-Provence, France.

Correspondance : Julie Chevalier, Aix Marseille Université, Site Schuman, 29 avenue Robert Schuman, 13621 Aix-en-Provence Cedex 01, France.

Courriel : juliechevalier3001@gmail.com

Texte reçu le 26 avril 2019 et accepté le 7 août 2019

http://www.bulletindepsychologie.net

Résumé : Notre article propose une nouvelle définition théorico-clinique du double en psychanalyse, dans ses liens aux processus adolescents. Pour cela, nous articulons d'abord les travaux de Rank $(1909,1932)$, sur le double et le héros, aux travaux de Freud $(1909,1913)$, sur le roman familial des névrosés et le mythe de totem et tabou, dans leurs articulations aux enjeux fraternels. Nous analysons ensuite le cas clinique d'une jeune femme de 16 ans, reçue pour un accompagnement thérapeutique, afin de montrer que le double constitue une figure du transfert, permettant à la patiente une actualisation de ses désirs fraternels, ainsi qu'une construction de sa romance amoureuse. Donc, le «double sororal et fraternel», que nous définissons, métamorphose le lien amoureux à l'adolescence.

\section{Functions of the double in adolescent romance in Rank and Freud}

Abstract: Our article proposes a new theoretical-clinical definition of the double in psychoanalysis, in relation to its links to adolescent processes. To do so, we first relate the works of Rank $(1909 ; 1932)$ on the double and the hero to the works of Freud $(1909 ; 1913)$ on the family romance of neurotics and the myth of totem and taboo, in terms of their links to fraternal issues. We then analyze the clinical case of a sixteen-year-old woman admitted for therapeutic support, to show that the double is a figure of transference, allowing the patient an update of her fraternal desires, as well as a construction of her romance. Therefore, the "sororal and fraternal double" that we define metamorphoses the bond of love in adolescence. 


\section{INTRODUCTION}

En psychanalyse, comme en littérature, le double est un concept riche et particulièrement polysémique. Notre article propose de cheminer en deux temps, d'abord en montrant comment la relation même du « père » de la psychanalyse Sigmund Freud avec l'un de ses premiers « fils » Otto Rank, peut en fait être lue comme une relation fraternelle, « en double ». Nous présentons ainsi comment la métapsychologie analytique de 1909 porte l'empreinte $\mathrm{du}$ thème et des processus du double, surtout en faisant surgir les enjeux œdipiens autour du sororal et du fraternel. Ensuite, nous proposons un moment clinique avec Valentina ${ }^{1}$, une adolescente, révélant comment les enjeux en double, dans le transfert, organisent sa romance amoureuse peuplée d'imagos héroïques sororales et fraternelles. L'essentiel de notre thèse peut se résumer de la manière suivante : en regard du lien unique entre Rank et Freud, nous soutenons que le lien amoureux se construit grâce à un quatuor d'imagos, le double et le héros, la sœur et le frère, ce pour quoi elles deviennent centrales dans les processus adolescents.

\section{RANK ET FREUD : FILIATION ET SYMÉTRIE}

Le lien unissant Freud et Rank semble singulièrement fort par son intense originalité. Remarquable par leurs textes qui s'enrichissent mutuellement, il suffit d'approcher l'œuvre freudienne pour constater que Rank est le seul à avoir été invité de façon aussi nette, nommément dans la quatrième édition de l'ouvrage freudien essentiel : L'interprétation des rêves» (Freud, 1900). Dans cet ouvrage, Freud a convié son ami pour l'écriture de deux chapitres sur le rêve, dans ses liens à la poésie (Rank, 1913a) et au mythe (Rank, 1913b), mais leur connivence intellectuelle était déjà apparue depuis la troisième édition de cet ouvrage exceptionnel, où Freud remerciait Rank pour ses corrections rigoureuses. Pourtant, les contributions personnelles de Rank ont été retranchées à partir de la huitième édition, pour donner suite à une rupture définitive entre les deux amis, au moment même de la parution de l'ouvrage Le traumatisme de la naissance (Rank, 1924). Dans cet ouvrage, Rank a articulé l'angoisse aux organes génitaux de la mère, ce par quoi la figure maternelle renaissait ainsi sous un jour nouveau au sein de la psychanalyse, tout en reléguant au second plan le fameux œdipe freudien.

Parallèlement, Rank a approfondi les fonctions mythiques de la figure du héros, en remerciant à son

1. Pour la présentation de notre cas clinique, tous les prénoms sont fictifs. tour Freud dans la première édition de son ouvrage «Le mythe de la naissance du héros» (1909), à l'intérieur duquel fut publié, en guise d'ouverture du chapitre III, l'intégralité du texte freudien «Le roman familial des névrosés» (Freud, 1909). C'est au travers de ces rencontres manuscrites que Rank et Freud ont alors influencé et soutenu leurs travaux respectifs, inscrivant les années 1908-1909 comme deux années charnières et fécondes. Un lien particulier s'est donc tissé entre les deux psychanalystes en favorisant, l'un pour l'autre, une forte créativité. Notre première hypothèse pose que le lien RankFreud s'est construit sous la forme d'une relation fraternelle, en double. En prolongeant cette formule, nous constatons qu'elle nous conduit à penser le double et le héros comme deux figures articulées dans des enjeux fraternels pour, aussi, conceptualiser ce que nous nommons le double sororal et fraternel. Notre deuxième hypothèse, que notre clinique vient soutenir avec le cas Valentina, se formule ainsi : le double sororal et fraternel permet de penser la construction du lien amoureux principalement dans ses liens aux processus adolescents.

Dans son ouvrage en partie historique, Safouan (2013) souligne l'écrivain «mauvais» qu'était Otto Rank, en contrepoint de son génie intellectuel, en citant les propres mots de Freud. Son génie traduisait une véritable honnêteté couronnée en partie d'un aspect énigmatique. Rank est alors reconnu en ses points créateurs et Freud confirme cette reconnaissance par sa franche invitation à occuper l'une de ses plus grandes œuvres analytiques, tout en scellant, en un sens, la naissance de la psychanalyse. Aussi, il est à noter que tous les deux proviennent de milieux précaires et qu'ils partagent une autre caractéristique commune autant étrange que familière, puisque Rank a échangé son nom «Rosenfeld» pour celui de «Rank», en signant là son intérêt pour l'un des personnages de la pièce de théâtre «Une maison de poupée » d'Ibsen (1879). Freud, quant à lui, a transformé son prénom « Sigismund Schlomo» pour celui de «Sigmund». Dans le récit de sa propre histoire personnelle, Rank, cadet de la famille, narre la liberté que son grand-frère Paul et lui ont pu nourrir face aux figures parentales, tout en admirant la droiture de son frère, peutêtre retrouvée en partie chez Freud, lui-même l'aîné de la famille. Rank semblait incarner, aux yeux des autres, un désir fondamental de vivre une existence de son propre choix, et cette ambition à exister en son nom, nous la saisissons alors en deux endroits précis : dans le nom qu'il choisit et dans le mythe de la naissance du héros. Au point que la naissance dans sa nomination prédestine celle de son œuvre. Il n'y a alors pas de hasard à ce que ce constat soit 
le point nodal entre le roman familial des névrosés et le mythe de la naissance du héros. Rappelons, d'ailleurs, que c'est le nom de Rank qui figure sur le certificat de décès d'Otto, au point que son père lui-même reçoit un nom de son propre fils ! (Safouan, 2013).

Les noms et prénoms métamorphosés résonnent pour nous comme un fort affront à la figure du père, et nous nous demandons s'il constitue l'une des clefs de la relation fraternelle entre Rank et Freud. Toujours est-il qu'il retentit à l'intérieur d'un autre mythe freudien, essentiel lui aussi, celui de la horde primitive, pareillement connu comme celui de meurtre du père (Freud, 1913). Nous avons en d'autres endroits travaillé les effets de noms-prénoms-signifiants dans la construction d'une relation en double (Chevalier, Bonnet, Gimenez, 2020), ce pourquoi, dans ce droit fil, nous considérons que, si Rank a pris une place importante au sein du cercle intellectuel de Freud, comptant déjà Fliess, Breuer, Adler, Jung, Abraham et Ferenczi, alors cela nous pousse à interroger d'une part, l'origine du double chez Rank, d'autre part, son articulation avec le sororal et le fraternel en psychanalyse. Le double serait-il donc un concept psychanalytique à revisiter?

\section{Le double : notion ou concept ?}

Si la notion est un terme visant à une généralisation et à une abstraction, le concept est un terme visant à s'articuler précisément à d'autres concepts dans un paradigme. Il devient alors légitime de se demander si le double est une notion ou un concept. Au sein des différents vocabulaires et dictionnaires de la psychanalyse, il n'y a pas réellement de référence conceptuelle au double. Si chez Laplanche et Pontalis (1967) son absence est nette, il n'en demeure pas moins que les travaux rankéens sont cités couramment chez Chemama (1993), Roudinesco (1997) ou Assoun (2009), bien que ce soit peut-être chez Delrieu (2001) qu'une certaine lisibilité apparaît davantage, depuis son occurrence au concept-notion de double chez Freud : « [...] le signe dont est affecté le double se modifie ; d'assurance de survie qu'il était, il devient l'inquiétant [unheimlich] avant-coureur de la mort. » (Freud, 1919, p. 237). Freud adresse-t-il ici un clin d'œil à son ami Rank ? La lecture critique et pointue de la littérature et des mythes offre à Rank (1932) la possibilité de saisir les motifs essentiels du double, c'est pourquoi il nous dit que chaque être ou chaque artiste, pour un souci d'exactitude, aux prises avec les enjeux complexes et étranges du double, entretient un rapport étroit au narcissisme, à l'égocentrisme et à une activité sexuelle dite « anormale ».
Leurs enjeux soutiennent le sujet dans une forme d'infatuation, tout en semblant similaires à ceux des processus adolescents que Gutton (2013) considère comme une période de création, où son créateur n'est autre que l'adolescent et l'adolescent sa création.

Le double toujours est paradoxal et ambivalent, de sorte à nous rappeler à nouveau certains concepts de la théorie freudienne. En 1915, Freud rédige Pulsions et destins de pulsions (Freud, 1915a) où nombreuses sont ses hypothèses dualistes qui éclosent en écho à la pensée rankéenne. Considérant la pulsion comme une excitation pour le psychique, sinon un psychique pulsionnel qui exciterait l'être, voire l'âme, Freud la définit comme un concept limite entre le psychique et le somatique, autrement dit, « comme le représentant psychique des excitations issues de l'intérieur du corps et parvenant à l'âme » (Freud, 1915a, p. 27). Rapprochant l'âme de l'inconscient, il devient soudainement étrange d'observer que Rank mit en exergue que l'âme fut l'image du corps, où l'ombre inséparable du sujet aurait été la première représentation «objective» de l'âme humaine et, donc, d'une croyance à la dualité de l'âme. En ce cas, peut-on supposer qu'il n'y a pas d'âme inconsciente chez Freud sans la signification d'une ombre immortelle chez Rank ? Pour qui, rapportons-le maintenant, le « jumeau paraît donc être l'homme qui, en venant au monde, a amené son Double immortel, c'est-à-dire l'âme » (Rank, 1932, p. 126).

Alors que Freud distingue trois polarités comme organisateurs de la vie psychique et pulsionnelle, la polarité biologique (dialectique activité-passivité), la polarité réelle (dialectique moi-monde extérieur) et la polarité économique (dialectique plaisir-déplaisir), nous pensons qu'y ajouter une polarité narcissique serait pertinent à la vue des propres dialectiques que la figure du double organise, à savoir le moi et le non-moi, l'amour et la haine et la vie et la mort. Afin d'appuyer nos propos, nous souhaitons insister sur une articulation entre les travaux de Rank et Winnicott, riche et utile pour travailler la figure du double. Nous y reviendrons, mais Rank (1932) part du constat que le frère cadet est la représentation la plus commune de la figure du double, celle par qui les enjeux du fraternel deviennent ainsi les plus lisibles. Ce constat étant pointé, il nous permet de repenser ce que Winnicott (1971) travaille de la première relation entre l'enfant et la mère, c'est-à-dire l'expérience d'un jeu de regards croisés, dont les frontières englobent à la fois le même et l'étranger, où l'un et l'autre, respectivement dans leurs regards, jubilent d'apercevoir chacun leur image dans les yeux de l'autre. C'est 
essentiellement aux abords de ce questionnement que l'adresse transférentielle entre en force dans la théorie winnicottienne, d'après laquelle nous considérons ainsi que l'enfant apprend d'abord à se construire en investissant sa mère ou son père comme un double sororal ou fraternel, étant entendu qu'aux instants critiques de cette relation, l'enfant ne sait rien de la réalité générationnelle qui le sépare de sa mère et son père. De cette façon, le double tend bien à questionner chez l'enfant l'existence d'un autre-que-moi (Winnicott, 1969), comme une entité à part entière, et que la destructivité et la haine permettent de faire exister, tout en recouvrant potentiellement certains des enjeux du complexe fraternel de Kaës (2008), à la fois quand il est question d'élaborer le corps imaginaire de la mère via les figures de la sœur ou du frère, avant qu'elles ne s'inscrivent classiquement dans le triangle rivalitaire et d'aboutir enfin à la construction du lien amoureux. Précisons que la destructivité et la haine structurent le fantasme chez Winnicott (1971), pour qu'il soit essentiellement un fantasme de meurtre à l'adolescence. Les processus adolescents seraient donc bien ceux par lesquels se réactualiseraient les désirs sororaux et fraternels infantiles. Ainsi, les processus adolescents organisent-ils la figure du double?

\section{Rank et Freud : des frères héroïques ?}

Le double peut être tout autant un ami qu'un ennemi, en variant au rythme d'une envie à jamais destructrice. Et c'est dans ce cas qu'il ne permet plus, comme le soulignait Freud (1910, 1912, 1918) avec audace, d'unir les courants tendre et sensuel, octroyant au sujet d'enfin jouir librement de sa capacité d'aimer, jamais mieux travaillée qu'aux limites de l'analyse de l'amour de transfert (Freud, 1915b). Rank et Freud, nous 1'avons souligné, se sont séparés en raison des hypothèses relatives au traumatisme de la naissance. Pourtant, il est curieux et étrange de retrouver, au sein des écrits freudiens sur la vie amoureuse, le passage suivant : « La naissance est aussi bien le tout premier danger pour la vie que le modèle de tous les dangers ultérieurs devant lesquels nous éprouvons l'angoisse, et vivre la naissance nous a vraisemblablement légué 1'expression-affect que nous appelons angoisse. " (Freud, 1910, p. 13). Le traumatisme de la naissance de Rank permettrait-il, finalement, de participer à la construction de la vie amoureuse ? Et le double à l'impérial amour de transfert?

Pour Safouan (2013), Rank était le fils préféré de Freud et, à ce titre, le plus haï. Mais selon notre lecture, il est possible de redistribuer leurs positions en fonction de leurs places fraternelles respectives.
Désormais, nous voyons surgir le couple fraternel, avec le tout de ce qui s'y joue, à savoir le désir d'inceste fraternel finissant de céder devant une rivalité meurtrière. L'ampleur de l'intensité de cette rivalité semble poindre au moment même de la fin tragique de la vie d'Otto Rank. Safouan (2013) nous la rappelle en repartant de son second mariage, le 31 juillet 1939, avec Estelle Buel, une jeune Américaine qui fut sa secrétaire à Paris. Il semblait qu'il connaissait avec elle des relations confiantes, lesquelles auguraient des années paisibles. Cependant, le sort en décida autrement. Freud meurt le 23 septembre 1939 et Rank le 31 octobre 1939, un mois après Freud. Leur relation fraternelle en double n'aurait pas permis sa propre métamorphose : Freud serait resté l'aîné envieux finissant avec violence d'assouvir ses pulsions sadiques et destructrices, et Rank le cadet héroïque embrassant son destin tragique en se privant lui-même d'un nouvel amour... Telle est, en tout cas, la thèse romantique de Safouan que nous embrassons dans notre propos, aussi renforcée par des rumeurs légendaires, puisqu'il se trouve que «Komisch » (Safouan, 2013) aurait été le dernier mot que l'on entendit prononcer par Rank. Il signifie drôle, bizarre, étrange, autant dire de parfaits synonymes du double, peut-être pour évoquer une fois dernière son ami Freud disparu un mois avant lui.

Dans son ouvrage de 1932, il est intéressant d'observer que Rank étudie 1'histoire légendaire de Don Juan en parallèle du double. Et ce d'autant plus qu'il fixe son attention sur les enjeux fraternels et amoureux de l'histoire, attendu qu'il est vrai que nous nous centrons régulièrement sur le personnage principal incarné par Don Juan, à défaut de prendre en considération l'existence de son fils Ramido. Ses relations amoureuses sont plus secrètes encore, car par quatre fois il se voit attiré par de jolies filles se trouvant être ses sœurs aimées. Ses relations amoureuses et tragiques se heurtent au désir d'inceste sororal et fraternel en révélant l'expression d'un amour absent de métamorphoses. Le destin héroïque de Ramido consiste donc dans une fin tragique, sinon dramatique, en tant qu'il se retrouve privé d'amour, où sourd ici comme un écho au destin tragique d'Otto Rank. C'est pourquoi le malheur du héros procède aussi de sa propre condition héroïque, amenant parfois avec elle l'incapacité de se défaire de son destin pour aimer un autre que lui et d'enfin devenir libre.

\section{LE DOUBLE SORORAL ET FRATERNEL}

Nous articulons préférentiellement le double aux processus adolescents dans leurs dimensions constructrices et non pathologiques, c'est pourquoi 
nous devons, pour mieux nous en détacher, revenir aux travaux psychanalytiques, qui jettent souvent un pont entre le double et les processus organisateurs de la psychose, sans prétendre être exhaustif à ce propos dans notre article. Classiquement le « double narcissique » est défini dans ses liens à la relation fétichique à l'objet (Baranes, 1995, 2002), à la relation duelle n'ouvrant pas sur une authentique triangulation (Gimenez, 2010) ou encore à 1'homosexualité primaire (Botella, Botella, 1995). Dans les conceptions analytiques générales, le double bascule régulièrement entre sa fonction de médiateur véritable entre soi et l'autre, ouvrant sur l'altérité, et sa fonction d'autre persécuteur au point d'être pensé essentiellement à travers le prisme défensif de la psyché. Conformément à cette position théorique, le double se réfléchit dans ses liens aux clivages du moi et de l'objet, puis de leurs installations, au point soit qu'il fige la psyché soit qu'il lui offre inversement de commencer à construire l'altérité sans être complètement le représentant original d'un tiers. À la suite, le double trouve ses spécificités extraordinaires à l'intérieur de la dynamique transférentielle, particulièrement dans les travaux de C. et S. Botella (1995). Cependant, leur conception ne repose pas encore sur l'idée du double comme un processus créatif et créateur de la continuité psychique du sujet, ce qui finira d'être nettement établi avec les travaux de Baranes (1995, 2002) et particulièrement de M'Uzan (2005). Désormais, le double se pense à l'entour de la transitionnalité et à la croisée des investissements narcissiques et objectaux. Ils rejoignent les positions théoriques de Roussillon (2009) et Jung (2012), lesquels vont nous permettre d'ouvrir sur les aspects adolescents, mais, en préalable à leur présentation, précisons encore notre définition du double sororal et fraternel.

Le double demeure une figure autour de laquelle gravitent de multiples synonymes. Ce sont, par exemple, ceux d'ombre, de reflet et de sosie parmi les plus répétitifs. Toutefois, ce sont l'ombre et son envers, le reflet, qui revêtent les spécificités les plus intéressantes en marquant nécessairement le double du sceau de la dualité. Rank (1932) différencie le double imaginaire et fantasmatique métamorphosé par l'ombre, le reflet ou le portrait, lequel fonde son expérience depuis le monde intérieur, en contrepoint du double réel, incarné principalement par le sosie, lequel fonde son expérience depuis le monde extérieur. En excès, l'ombre et le reflet deviennent alors deux représentations différentes de l'âme humaine : l'ombre est une âme noire et haineuse, annonciatrice de la mort, le reflet une âme claire et amoureuse, annonciatrice de la vie. Le double est donc tout autant gardien de la vie qu'annon- ciateur d'une mort narcissique toujours à venir, où l'enjeu psychique du dualisme amour-haine est apothéotique.

Dans son commentaire de « La nuit de décembre » (Musset, 1835), Rank (1932) observe que Musset représente le double grâce à une ombre fraternelle, ressemblant au héros comme un frère et renforçant son idée de considérer essentiellement le double, depuis les spécificités de l'imago du frère cadet devenant l'image vivante du moi fraternel et amenant toujours avec elle la rivalité, voire la haine fraternelle. L'affrontement moïque du sujet à son double le conduit sur les affres singulières d'un désir ô combien puissant : le désir d'immortalité. Est alors un héros celui qui se dresse contre la mort (Bonnet, Pechikoff, Petit, 2014) sinon la mort de son propre moi. Antérieurement, nous évoquions le couple fraternel Rank-Freud à l'intérieur duquel surgissent des désirs incestueux et meurtriers, lesquels nous permettent maintenant d'apprécier la différence majeure entre le gémellaire et le simple fraternel d'un point de vue fantasmatique. Pour cela, nous repartons du mythe du héros rankéen, afin de mettre en lumière que le gémellaire trouverait son origine dans l'identique en imposant particulièrement aux jumeaux de s'interroger sur ce que l'un a pu dérober à l'autre. Autrement dit, le lien gémellaire au sein duquel la dérobade s'inscrit intime la conviction, à l'un comme à l'autre des jumeaux, d'avoir été lésé d'une chose, en réalité la chose sexuelle, en les menant à un désir de vengeance, voire, en dernier ressort, à un désir de meurtre. La violence de ce désir atteint son apogée quand survient la rencontre avec une figure sexuelle, sinon érotisée, où l'un des jumeaux encourt le risque d'une exclusion définitive. C'est pourquoi, ici, la figure amoureuse détermine et provoque entre les jumeaux la « pulsion meurtrière-fraternelle » (Chevalier, Bonnet, Gimenez, 2018). En son contrepoint, nous situons le fraternel à la lisière du désir d'inceste poussant les sœurs et les frères à s'unir, et qui apparaissait déjà dans le roman familial des névrosés freudien comme son leitmotiv. Cela nous rappelle deux choses : la première est la version du mythe freudien de totem et tabou, où les frères font alliance tous ensemble, sans exception, afin d'accéder aux sœurs en tuant le père, tout en montrant que le premier meurtre de la civilisation ne procède pas d'une quête héroïque pour posséder la mère, mais, au contraire, d'une quête héroïque pour posséder la sœur. Et la seconde est celle que nous rappelle Rank (1909) à propos de la structure du fantasme, sa nature se concluant soit par la vengeance et, pour ainsi dire, le meurtre, soit par le sauvetage et, pour ainsi dire, l'inceste. À cette idée fait écho ce que G. 
Bonnet (2013) met au jour à propos de l'existence de deux formes d'héroïsme, où, de l'une à l'autre, nous retrouvons les désirs fraternels meurtriers et incestueux : la première incarne le héros dans une lutte à mort et la deuxième dans un combat de tous les instants pour sauver sa vie. En définitive, nous soutenons que le double sororal et fraternel recouvre le désir de meurtre gémellaire et le désir d'inceste sororal, tous deux mis en scène dans les mythes et les légendes, en imposant au héros de tuer son jumeau ou de sauver sa sœur ou son frère. Désormais, nous pouvons préciser que le double sororal et fraternel est celui par lequel le sujet s'affronte aux désirs fraternels incestueux et meurtriers, des premières relations en doubles, fantasmatiques ou réelles, avec une figure investie comme une sœur ou un frère.

\section{Le double et les processus adolescents}

Dans les travaux psychanalytiques, nous retrouvons souvent le double articulé aux enjeux du narcissisme, et particulièrement à l'endroit de l'instance du moi-idéal depuis laquelle, systématiquement, l'on voit surgir soit le double narcissique soit le lien homosexuel. Avec le moi-idéal, le double reste enclos dans une structure duelle où le tiers, et en ce sens l'autre, serait en partie manquant en n'aboutissant pas à la rencontre amoureuse telle qu'elle est conçue par Freud et Winnicott. Cependant, l'un de nous a déjà montré que l'ami et l'amoureux sont choisis par les adolescents comme des doubles ou des semblances (Bonnet, Pechikoff, 2007). D'autant plus que la particularité de ce choix amoureux s'inscrit dans des triangles amoureux : un amoureux entre deux amis, un ami entre deux amoureux. Le double sororal et fraternel que nous conceptualisons englobe l'ensemble de ces triangles amoureux formant une combinatoire, ce par quoi il forme une union avec les figures de la sœur et du frère cadets, élues comme deux figures idéales.

La sœur et le frère cadets prennent une place essentielle dans la galaxie des imagos gravitant autour du double et du héros, en devenant leurs images vivantes. En plus d'être les premiers doubles du sujet, les cadets sont aussi ceux que les figures d'autorité appellent pour incarner les héros familiaux. Au sein de la fratrie, leur position les conduit, en effet, à dépasser l'ordre familial établi en étant le préféré des figures parentales, sorte de rappel de toute la littérature picaresque. Et, en tant que doubles préférés, c'est alors à la cadette et au cadet que tous les désirs incestueux et meurtriers de l'ensemble de la fratrie (ou des pairs) reviennent. Pour les adolescents en analyse, les instances idéales de la sœur cadette préférée et du frère cadet préféré offrent souvent l'agencement et le déploiement du «roman adolescent» (Bonnet, Pechikoff, 2011), au sein duquel ils construisent en partie une multitude d'imagos héroïques, en rappelant d'ailleurs le « héros à la cicatrice » (Bonnet, 2016) et la « fonction » de transition (Camus-Donnet, 2008) et de médiation du fraternel entre le sujet et son autre amoureux. Les ami(e)s des adolescents apparaissent souvent dans leurs relations comme des sœurs ou des frères de cœur, selon le modèle de messagers envers leurs objets d'amour. Par ces entrelacs variés, le double sororal et fraternel apparaît donc comme une figure du transfert, construite par l'adolescent et le clinicien, se constituant comme le lieu d'un investissement amoureux ou haineux, non pas limité à un ordre duel, mais ouvrant sur une structure triangulée.

En ayant montré comment le double est articulé dans des enjeux fraternels, nous sommes en mesure d'affirmer qu'il est un compagnon idéal de l'adolescent dans sa quête amoureuse. Mais que cela vient-il préciser des processus adolescents ? Les processus adolescents exercent simultanément tout un remaniement psychique imposant à l'adolescent de sortir vainqueur des épreuves du pubertaire (Aubray, Agostini, 2013), tel qu'il est théorisé par Gutton (2013), ainsi que tout un remaniement narcissique. Ces remaniements lui imposant, cette fois-ci, de sortir héroïque des enjeux du complexe fraternel, pour essentiels qu'ils sont dans la construction du lien amoureux. Quand Freud écrit Pour introduire le narcissisme en 1914, c'est ici qu'il précise que le sujet a deux objets sexuels originaires : la mère et lui-même. La mère qui, d'après nos propos précédents, fonctionnerait à la manière d'une figure sororale ou fraternelle aux prémices de leur relation. Les figures fraternelles en double deviennent ainsi centrales chez l'enfant, pour construire d'abord un autre-que-moi, puis, chez l'adolescent, pour construire un autre amoureux, étant entendu que Freud, dans ses Contributions à la psychologie de la vie amoureuse $(1910,1912,1918)$, souligne que la capacité d'aimer un autre serait supérieure à celle d'être aimé. Donc l'état amoureux s'exerce dans un au-delà de soi-même.

Dans ses Contributions à la psychologie de la vie amoureuse, Freud adosse le choix d'objet de l'homme à un clivage brutal entre la mère et la putain, la première étant aimée à l'endroit où la deuxième est désirée. Ce clivage ne permet pas d'aboutir à l'union essentielle des courants tendre et sensuel accordant au sujet de faire de lui un homme cultivé, à la capacité d'aimer. C'est ainsi que l'homme à l'amour clivé ne choisit pas une femme libre, mais, au contraire, une femme unie à un autre 
homme, de sorte à faire valoir d'abord des droits de propriété. À la suite de quoi, il finit de désirer une autre femme, dont la fidélité et la fiabilité autorisent le doute, pour l'investir seulement comme un objet. Bien que la première condition s'observe souvent indépendamment de la seconde, on ne saurait repousser l'évidence que la seconde finit par s'articuler à la première : à la femme-possédée s'adjoint la femme-objet. Quand l'homme et la femme sont aux prises avec ces relations, il va de soi que nous pouvons soutenir qu'ils ne connaissent ni la faculté d'aimer librement, ni l'état amoureux, attendu que, dans ces conditions, la tendresse procéderait de la possession et le sensuel de l'objectalisation.

Dans l'ordre de ce clivage émane la question du rabaissement de l'objet sexuel. L'homme fait preuve d'une impuissance psychique, fréquemment en raison d'une fixation incestueuse à la mère ou à la sœur. Le courant tendre, le plus ancien, s'entrechoque avec le courant sensuel surgissant à l'âge de la puberté, ce pourquoi leur réunion constituerait un enjeu majeur des processus adolescents dans leur potentialité à réactualiser tout autant les enjeux œdipiens que les enjeux fraternels. Cependant, toute la sensualité dans l'inconscient peut se rattacher à des objets incestueux avec un effet double : l'objet sexuel est rabaissé à l'endroit où l'objet incestueux est surestimé. Il semble donc que, pour être libre d'aimer et de renoncer à une quête illusoire d'un objet rabaissé ou d'un objet narcissique, le sujet doive s'affronter pleinement à ses désirs incestueux, qu'ils se présentent du côté de la mère ou de la sœur. Pour partie, les processus adolescents incarnent le lieu de ce combat inévitable dans la construction du lien amoureux, ce pourquoi nous terminons en formulant que le double, car il est articulé dans des enjeux fraternels traitant d'inceste et de meurtre, est celui par lequel l'adolescent est à même de travailler à fondre en un seul fleuve les courants tendre et sensuel.

\section{Valentina et ses figures érotiques/héroïques}

Afin d'articuler nos hypothèses à la clinique, nous relatons des fragments de la rencontre de l'un de nous avec Valentina, une jeune femme de 16 ans accueillie dans un foyer d'enfance. Dans ses relations, la mort se tisse à merveille à l'amour et nous sommes toujours frappés par son corps squelettique. Nous l'imaginons façonné par la violence à laquelle elle se confronte en permanence. Seule la blondeur de ses cheveux illumine son visage, donnant un éclat perçant au bleu de ses yeux. C'est ainsi que, tiraillée face au choix de continuer ou finir sa relation amoureuse, Valentina vient demander notre aide. Sa relation amoureuse est ponctuée par les coups violents et multiples que son partenaire et elle se renvoient de façon mutuelle et, durant les différentes séances où nous la recevons, Valentina nous fait le récit de ses aventures adolescentes mêlant la sexualité à la violence, la drogue à la mort. Qu'il soit question de ses histoires amoureuses ou d'amitié, elles se retrouvent toujours bornées par des enjeux autour du double comme pour s'affronter à sa continuité narcissique ainsi qu'à l'altérité radicale de l'autre.

Valentina.- Vous savez, j'ai repris contact avec mon frère, je suis trop contente !

Nous.- Mais je ne savais pas que tu avais un frère...

Valentina.- Si, un grand-frère, il s'appelle Valentino. On était supers proches. Bah! déjà rien que nos prénoms, je m'appelle Valentina et lui Valentino. Valentina Valentino. Le pire dans tout ça, c'est qu'il nous arrive les mêmes choses. Moi j'étais enceinte et j'ai avorté alors que mon copain ne voulait pas. Et lui, sa copine est enceinte et ne veut pas avorter alors que lui veut qu'elle avorte. C'est fou quand même qu'on vive les mêmes choses.

Nous.- Tu dis que vos situations se ressemblent, à la manière de vos prénoms. Qu'en penses-tu ?

Valentina.- J'sais pas trop, entre moi et Valentino en même temps ça a toujours été particulier. On a arrêté de se parler car il a cru que j'avais couché avec $\mathrm{j}$ 'sais pas qui.

Nous.- Ce que tu dis là me fait penser à une réaction que ton copain a déjà eue envers toi.

Valentina.- Ouais c'est vrai que c'est bizarre, bon en même temps c'est particulier la relation entre moi et Valentino! D'ailleurs c'est énorme, vous savez quoi, mon grand-frère est né le 12 novembre et c'est la même date à laquelle a commencé mon histoire avec mon copain, c'est un truc de fou !

«Valentina-Valentino» nous dit Valentina de façon symphonique, où nous pensons ici comme au titre d'une pièce de théâtre à la manière de Molière, promettant de lever le voile d'un secret tout aussi énigmatique pour nous qu'il l'est, à ce moment-là, pour elle. Les destins fraternels des désirs incestueux et meurtriers entre Valentina et son frère aîné, Valentino, se réactualisent aujourd'hui dans sa relation amoureuse gouvernée par la haine et la violence. L'idée répétée de trouver dans les dires de Valentina la naissance articulée à la mort par le biais de l'avortement pourrait s'entendre de différentes façons. La question du féminin paraît se confondre avec celle du maternel, au point que nous nous remémorons la jubilation de Valentina quand, enceinte, elle se sentait femme. Mais, alors qu'elle 
articule son idée à la figure de son frère-aîné, nous y entendons également l'imago de « la mère-aux frères-et-sœurs » de Kaës (2008), laquelle permet au sujet l'exploration du corps maternel imaginaire via les frères et les sœurs. Une fois cette variante du fantasme traversée, il s'avère que la deuxième donne à voir l'accouplement des frères et des sœurs afin de se séparer, désormais, de la figure maternelle et de procéder ainsi à la construction du lien à un autre. Cela se repère en partie quand Valentina évoque la rupture conflictuelle avec son frère en raison de l'un de ses rapports sexuels prétendus. Il semblerait que les désirs incestueux ne se soient pas entièrement résolus afin de permettre, à l'un comme à l'autre, d'investir un objet d'amour porteur de leur narcissisme de vie. C'est ce que semble nous révéler Valentina en fin de séance, quand ellemême, surprise, associe la date de la naissance de son frère à celle de sa relation amoureuse.

Dans la dynamique transférentielle, Valentina parvient à ressentir la ressemblance bizarre entre sa présente relation amoureuse et son ancienne relation fraternelle. Son «c'est bizarre» entre en résonnance avec l'inquiétante étrangeté de Freud (1919), que la rencontre avec un double peut susciter. En tant qu'il est articulé à l'inceste et au meurtre, le double ici est accompagné par le meurtre fantasmatique d'un enfant à venir, car il est vécu comme le fruit d'un accouplement incestueux, ramenant toujours avec lui l'horreur de l'inceste (Stein, 1971) et, en dernier ressort, l'horreur de la mort. Ce que le double sororal et fraternel met aussi en évidence, c'est l'affrontement de l'adolescent à ses figures érotiques héroïsées, dès lors qu'elles s'unissent aux imagos idéales de la sœur et du frère. Nous pouvons envisager, avec Valentina, que les enjeux du double, dans les processus adolescents, se romancent au rythme de trois batailles : du moi narcissique pour rencontrer l'autre, de l'inceste et du meurtre fraternels pour rencontrer l'autre amoureux, de la vie et de la mort tragique et héroïque pour devenir libre de son destin.

Considérons maintenant comment, dans un autre moment clinique, la valeur de ces éléments prend à nouveau forme dans la romance amoureuse de Valentina. Outre sa relation avec son partenaire masculin, il y a également toutes ses relations passionnelles, à la fois avec Césarina, sa meilleure amie, et les femmes. Leurs apparitions successives nous conduisirent un jour à lui formuler, dans le transfert, la question qui suit :

Nous. - N'as-tu jamais imaginé ou pensé, Valentina, que tu aimerais peut-être vivre une relation amoureuse avec une femme?
Valentina. - Bah si ! Moi si Césarina avait voulu, j'aurai vécu un truc avec elle. En fait, si je devais avoir une relation amoureuse avec une femme, j'aimerais que ce soit complètement caché, que ce soit un secret. D'abord, j'aimerais que ça commence par une histoire d'amitié et j'aimerais aussi que ce soit vraiment une histoire entre deux femmes.

Nous.- Souvent tu me parles de Césarina, comment est-elle?

Valentina._- Elle est comme moi, elle est blonde, en fait elle est comme moi sauf qu'elle est plus petite. C'est moi en miniature. C'est drôle, mais je me sens attirée par les femmes parfois. J'sais pas, les femmes c'est plus joli à regarder qu'un homme.

Nous.-Quelles femmes regardes-tu ?

Valentina. - À chaque fois, on m'dit, allez mange un peu Valentina, parce que je suis maigre. J'ai pas de formes. Moi les femmes que je trouve belles et que je regarde, ce sont les femmes brunes qui ont de beaux seins et de belles fesses et qui ont les yeux verts. J'aimerais bien avoir les yeux verts.

À la suite de cet intense moment clinique, les mots de Valentina nous évoquent nos propres yeux verts et nous ramènent au souvenir de notre première séance, durant laquelle elle dessina le portrait d'une femme enveloppée d'une grande chevelure marron à l'image de notre propre coiffure. Nous apparaissons dans le transfert avec Valentina comme une figure à partir de laquelle elle paraît s'affronter, dans une sorte de renversement, au féminin comme au maternel. Nous l'avons souligné, pour nous la mère fonctionnerait comme une figure sororale ou fraternelle offrant à l'enfant de se séparer d'elle, c'est pourquoi l'on y entend aussi comment Valentina interroge le lien amoureux en revenant à l'exploration de son corps imaginaire en nous investissant comme un double dans le transfert. Dans le complexe fraternel, les frères et les sœurs constituent des appendices du corps de la mère (Kaës, 2008), ils permettent aussi bien l'exploration du corps maternel que le propre corps de l'enfant. C'est pourquoi il ne serait donc pas uniquement question de relation à un objet primaire, dans son articulation au lien homosexuel, ce qui nous semble constituer des « catégories » fermées. Nous leur préférons le concept d'être-au-monde (fidèle à celle du Dasein de Binswanger en 1971), pour travailler la question que nous nommons l'être-à-l'autre, dit autrement, le lien amoureux.

Cependant, une lecture supplémentaire est possible puisque la diversité des récits de Valentina, structurant sa romance amoureuse, actualise un jeu de prénoms, soutien de son activité de pensée et de 
représentation, les prénoms et les noms qui étaient aussi des éléments essentiels dans notre lecture du lien Rank-Freud. Ce jeu est, pour nous, le signe d'un « transfert en double » (Chevalier, Bonnet, Gimenez, 2018) combinant plusieurs triangles amoureux avec, comme figure organisatrice stable, la figure du frère ou de la sœur. Au croisement des dires de Valentina s'entremêlent désormais les enjeux du lien amoureux aux éléments du lien sororal. Le prénom de son amie «Césarina » nourrit une proximité phonétique et visuelle importante avec le prénom de sa petite sœur « Catharina », aussi blonde et plus petite que Valentina, devenant cette fois-ci vivante dans nos propres associations. Césarina, « miniature " de Valentina, devient un analogon psychique et inconscient de l'imago de sa sœur cadette dans le transfert, au sein duquel se renforce l'importance de signifiants, comme les prénoms pour la lecture et l'analyse de relations en double. Lorsque Valentina évoquait précédemment sa relation amoureuse avec son partenaire masculin, la figure du frère aîné apparaissait clairement dans son discours, pour penser le meurtre fraternel fantasmatique dans son association à l'avortement, ainsi que l'accouplement incestueux, amenant avec lui l'horreur de l'inceste et de la mort. Quand Valentina rapporte maintenant sa relation amoureuse fantasmatique avec Césarina, la figure de la sœur cadette apparaît dans les associations de la clinicienne, au point de permettre un jeu de renversement entre féminin et maternel. La figure de la sœur permet d'abord d'interroger le féminin par le lien amoureux à une figure féminine, puis la clinicienne, apparaissant à la suite comme une autre figure sororale idéale, permet à Valentina de travailler le rapport à son propre corps et, dans le même temps, au corps maternel. Au terme de cette analyse nous formulons donc que les processus adolescents sont bien des processus fondateurs des constructions amoureuses : le double structure des triangles amoureux et s'articule aux enjeux du fraternel, tels que le meurtre, l'inceste et les métamorphoses du corps imaginaire.

\section{CONCLUSION}

Nous avons souligné, au début de notre texte, comment la relation en double entre Rank et Freud fut remarquable dans l'entrelacs de leurs apports mutuels dans les textes et, surtout, à quel point elle était repérable non pas comme un lien PèreFils, mais bien comme empreinte d'enjeux fraternels. Que le lien Rank-Freud soit articulé dans des enjeux fraternels, nous a permis d'explorer comment, du mythe du héros jusqu'au roman familial des névrosés, ce sont les désirs incestueux sororaux et fratricides qui se révèlent riches pour travailler le lien amoureux. Depuis ce constat entre Héros rankéen et Edipe freudien nous dégageons toute l'importance des figures de la sœur cadette et $\mathrm{du}$ frère cadet dans un roman familial. Tous deux deviennent des figures idéales dont les enjeux désirants infantiles sont remarquables et deviennent intenses dans les processus adolescents, voire dans un roman adolescent. Dans la lignée de la pensée psychanalytique, et non pas développementale, nous posons que les processus adolescents sont le lieu de tout un remaniement narcissique requérant de penser l'autre amoureux en s'affrontant à ses désirs fraternels incestueux et meurtriers.

La clinique avec Valentina et sa romance, nous a semblé forte des enjeux du double au sein de la dynamique transférentielle, particulièrement pour montrer l'alliance entre l'être et l'amour dans ses processus adolescents. Ainsi, nous soutenons que les processus adolescents réactualisent les désirs sororaux et fraternels infantiles et ce électivement au travers de la figure du double. La conséquence clinique (et Valentina en révèle les arêtes vives), ainsi que conceptuelle, n'est autre que le double sororal et fraternel constitue une figure du transfert, construite par l'adolescent et le clinicien. Cette importance du double sororal et fraternel ne saurait se dissocier de la qualité particulière que revêtent la sœur cadette ou le frère cadet. Ce sont, en quelque sorte, des prototypes du double devenant le lieu d'investissements amoureux ou haineux, qui ouvrent sur une structure triangulée.

Enfin, nous soutenons que le double ne s'envisage plus comme une figure symptomatique d'une psychopathologie des psychoses, mais bien plutôt comme un opérateur structurant et éminemment articulé aux processus adolescents. Et, dans son alliance avec les enjeux fraternels traitant d'inceste et de meurtre, le double devient le moyen, pour l'adolescent, de travailler à harmoniser étroitement les courants tendre et sensuel ; c'est ainsi qu'il structure une véritable combinatoire de triangles amoureux, en révélant de multiples enjeux $\mathrm{du}$ fraternel tels que le meurtre, l'inceste et les métamorphoses du corps imaginaire. Les enjeux métapsychologiques, ainsi que transférentiels, de la figure du double ouvrent toujours à de nombreuses questions dont nous venons d'esquisser les lignes de force dans notre analyse. 


\section{RÉFÉRENCES}

Assoun (Paul-Laurent).- Dictionnaire thématique, historique et critique des auvres psychanalytiques, Paris, Presses universitaires de France, 2009.

Aubray (Marie-Christine), Agostini (Dominique).Travail du héros, Adolescence, 31, 2, 2013, p. 299-312.

BARANEs (Jean-José).- Double narcissique et clivage du moi, Revue française de psychanalyse, 1995, p. 39-53.

BARAnes (Jean-José).- Penser le double, Revue française de psychanalyse, 66, 5, 2002, p. 1837-1843.

BINSWANGER (Ludwig).- Introduction à l'analyse existentielle, Paris, Éditions de minuit, 1971.

Bonnet (Christian).- Le mythe du « héros à la cicatrice » : une structure idéologique adolescente ou « quand le héros affronte la mort de la mort », Revue de psychothérapie psychanalytique de groupe, 67, 2, 2016, p. 157-166.

Bonnet (Christian), Pechikoff (Stéphanie).- À l'ami à l'amour, Adolescence, 61, 3, 2007, p. 561-517.

Bonnet (Christian), Pechikoff (Stéphanie). - Roman adolescent et scènes pubertaires, Adolescence, 2011, p. 787-800.

Bonnet (Christian), Pechikoff (Stéphanie), Petit (Laetitia).- De l'exposition du héros aux blasons du corps, Topique, 2014, p. 25-38.

BonNET (Gérard).- Du héros tragique au héros ordinaire, Adolescence, 31, 2, 2013, p. 313-326.

Botella (César), Botella (Sara).- La dynamique du double : animique, auto-érotique, narcissique. Le travail en double, Revue française de psychanalyse, 1995, p. 65-82.

CAmus-Donnet (Danièle).- La fonction fraternelle, Revue française de psychanalyse, 72, 2, 2008, p. 409-417.

Chemama (Roland).- Dictionnaire de la psychanalyse : dictionnaire actuel des signifiants, concepts et mathèmes de la psychanalyse, Paris, Larousse, 1993.

Chevalier (Julie), Bonnet (Christian), Gimenez (Guy).Le double héroïque : entre mythe et roman, Adolescence, 36, 2, 2018, p. 379-388.

Chevalier (Julie), Bonnet (Christian), Gimenez (Guy).L'amour du transfert : " un autre même », Cliniques méditerranéennes, 2020, sous presse.

Delrieu (André).- Sigmund Freud : index thématique, Paris, Économica, 2001.

Freud (Sigmund).- L'interprétation des rêves [1900], Paris, Presses universitaires de France, 1987.

Freud (Sigmund).- Le roman familial des névrosés [1909], Paris, Payot, 2014.

FreUd (Sigmund).- Contributions à la psychologie de la vie amoureuse [1910, 1912, 1918], Paris, Presses universitaires de France, 2011.

Freud (Sigmund).- Totem et tabou [1913], Paris, Payot, 2001.

Freud (Sigmund).- Pour introduire le narcissisme [1914], Paris, Payot, 2012.

Freud (Sigmund).- Pulsions et destins de pulsions [1915a], Paris, Payot, 2017.

FREUD (Sigmund).--Observations sur l'amour de transfert [1915b], dans Freud (S.), La technique psychanalytique, Paris, Presses universitaires de France, 1953, p. 116-130.

Freud (Sigmund).- L'inquiétante étrangeté et autres essais [1919], Paris, Gallimard, 1985.

Gimenez (Guy).- Halluciner, percevoir l'impensé : approche psychanalytique de l'hallucination psychotique, Louvain-la-Neuve, De Boeck Supérieur, 2010.

Gutton (Philippe).- Le pubertaire, Paris, Presses universitaires de France, 2013.

IBSEN (Henri).- Une maison de poupée [1879], Paris, Le livre de poche, 1968.

JuNG (Johan).- Le double transitionnel. Trajectoire identitaire et organisation réflexive, thèse de doctorat, Université Lumière Lyon 2, 2012.

KAËs (René).- Le complexe fraternel, Paris, Dunod, 2008.

Laplanche (Jean), Pontalis (Jean-Bertrand).- Vocabulaire de la psychanalyse, Paris, Presses universitaires de France, 1967.

M'Uzan (Michel de).- Le jumeau paraphrénique ou aux confins de l'identité, dans Aux confins de l'identité, Paris, Gallimard, 2005, p. 14-40.

Musset (Alfred de).-Les nuits : Nuit de mai, Nuit de décembre, Nuit d'août et Nuit d'octobre, Paris, C. Meunier, 1835.

RANK (Otto).- Le mythe de la naissance du héros [1909], Paris, Payot \& Rivages, 1983.

RANK (Otto).- Rêve et création poétique [1913a], dans Freud (S.), L'interprétation des rêves, Paris, Presses universitaires de France, 1950, p. 379-405.

RANK (Otto).- Rêve et mythe [1913b], dans Freud (S.), L'interprétation des rêves, Paris, Presses universitaires de France, 1950, p. 406-420.

RANK (Otto).- Le traumatisme de la naissance [1924], Paris, Payot, 1990.

Rank (Otto).- Don Juan et le Double [1932], Paris, Payot, 2001.

RoudINESCO (Élisabeth).- Dictionnaire de la psychanalyse, Paris, Fayard, 1997.

RoussiLlon (René).- Le partage de l'affect et la réflexivité par l'homosexualité primaire « en double », dans $L e$ transitionnel, le sexuel et la réflexivité, Paris, Dunod, 2009, p. 103-120.

SAFounn (Moustapha).- La psychanalyse. Science, thérapie et cause, Paris, Gallimard, 2013.

Stein (Conrad).- L'enfant imaginaire, Paris, Denoël, 1971.

WinnicotT (Donald Woods).- L'usage de l'objet et le mode de relation à l'objet au travers des identifications [1969], dans Winnicott (D. W.), La crainte de l'effondrement et autres situations cliniques, Paris, Gallimard, 2000, p. 231-242.

Winnicott (Donald Woods).- Jeu et réalité, Paris, Gallimard, 1971. 